\title{
High-gain erbium-doped fiber amplifier incorporating a double-pass amplification technique as a preamplifier
}

\begin{abstract}
We present a high-gain erbium-doped fiber amplifier to be utilized as a preamplifier. A double-pass amplification technique is used in the first-stage amplifier together with a tunable bandpass filter. The secondstage amplifier is a counter-pumped configuration and another tunable bandpass filter is utilized to filter out amplified spontaneous emission from the firststage amplifier. This design is able to produce a high gain of $55.6 \mathrm{~dB}$ and a noise figure of $6.02 \mathrm{~dB}$ at $1530 \mathrm{~nm}$ with a signal power of $-45 \mathrm{dBm}$. The receiver sensitivity measurement shows that the proposed amplifier improves the minimum detectable power from -33.7 to $-40.8 \mathrm{dBm}$ for a bit-error rate of $10-11$ at $155 \mathrm{Mbps}$.
\end{abstract}

